\title{
Hepatitis C virus genotype 4-infection and interferon-free treatment in Egypt
}

\author{
Tatsuo Kanda $^{1}$ (D) Shunichi Matsuoka ${ }^{1} \cdot$ Mitsuhiko Moriyama $^{1}$
}

Received: 8 May 2018/Accepted: 12 June 2018/Published online: 10 July 2018

(C) Asian Pacific Association for the Study of the Liver 2018

Keywords HCV · Genotype 4 - DAA · SVR · Thyroid-stimulating hormone

$\begin{array}{ll}\text { Abbreviations } \\ \text { HCV } & \text { Hepatitis C virus } \\ \text { GT } & \text { Genotype } \\ \text { HCC } & \text { Hepatocellular carcinoma } \\ \text { DAA } & \text { Direct-acting antivirals } \\ \text { SVR } & \text { Sustained virological response } \\ \text { TSH } & \text { Thyroid-stimulating hormone }\end{array}$

In interferon-free treatment era, therapeutic regimens and sustained virological response (SVR) rates differ among different hepatitis C virus (HCV) genotypes (GTs) with either interferon-including or interferon-free treatments. The estimated rates of HCV infection in Egypt is around $14.7 \%$, and the predominant HCV GT is GT4, where GT4a, GT4b, GT1 and GT3 represent 63, 30, 6 and 1\% of cases, respectively [1]. Recently, the results of treatment with combinations of direct-acting antivirals (DAAs) for $\mathrm{HCV}$ GT4 Egyptian patients have been reported [2-6]. Almost all of these regimens could lead to more than $95 \%$ or more than 90\% SVR rates, respectively, in the following patients: "easy-to-treat" patients who were treatment-naïve and did not have cirrhosis but had compensated liver biochemical parameters or "difficult-to-treat" patients who had previously failed interferon therapy with or without DAAs or an interferon-free combination, regardless of fibrosis stage, and had cirrhosis.

Daclatasvir (DCV) is a pangenotypic nonstructural protein 5A (NS5A) inhibitor [7]. Sofosbuvir (SOF) is a nucleoside inhibitor of $\mathrm{HCV}$ NS5B with pangenotypic potency [8]. The combination of SOF/DCV with or without ribavirin makes up one of the pangenotypic regimens.

Tatsuo Kanda

kanda.tatsuo@nihon-u.ac.jp

1 Division of Gastroenterology and Hepatology, Department of Medicine, Nihon University School of Medicine, 30-1 Oyaguchi-kamicho, Itabashi-ku, Tokyo 173-8610, Japan
Shiha et al. [2] investigated the efficacy and safety of 12 or 24 weeks of $400 \mathrm{mg} /$ day SOF plus $60 \mathrm{mg} /$ day DCV, with or without 800-1000 mg/day ribavirin, in HCV GT4 Egyptian patients. Although this was an open-label observational study, it includes a total 1168 patients: 385 patients with cirrhosis and 1063 treatment-naïve patients [2]. The authors observed rates of 96 and $93 \%$ SVR at 12 weeks (SVR12), respectively, with 12- and 24-week combinations of SOF/DCV with or without ribavirin, both in naïve and treatment-experienced patients with or without cirrhosis.

Shiha et al. [2] demonstrated that combinations of SOF/ DCV with or without ribavirin for 12 or 24 weeks was effective and safe for chronic HCV GT4 "real-world" patients. SVR rates were higher for patients with no cirrhosis although APASL, AASLD or EASL HCV guidelines do not recommend SOF/DCV for HCV GT4 patients without cirrhosis [8-10]. SVR rates for the combination of SOF/DCV were almost similar to those for the combination of the HCV NS5A inhibitor ledipasvir (LDV)/SOF with or without ribavirin for HCV GT4 [2], demonstrating a SVR12 of $98 \%$ in treatment-naïve patients and 94-98\% in treatment-experienced patients [5]. The presence of cirrhosis was associated with lower SVR12 rates in all treatment groups [2]. The authors also observed that the addition of ribavirin to the 24-week combination of SOF/ DCV was associated with a significant increase in the SVR12 rate in the treatment-experienced group [2], supporting APASL recommendation [8], although the mechanism of action of ribavirin remain unknown [11]. During SOF/DCV treatment, the most common adverse events were insomnia, fatigue, headache, anemia and cough, and no treatment-related serious adverse events were observed [2].

Abdel-Moneim et al. [3] also analyzed data from a large cohort of $946 \mathrm{HCV}$ GT4 Egyptian patients treated with a 12-week combination of SOF/DCV with or without 
ribavirin. HCV GT4a, GT4 m, GT4n, and GT4o showed rates of 76, 5, 7 and 12\%, and IL28B GTs CC, CT and TT of 19,54 and $27 \%$, respectively. According to the FIB-4 score, the authors defined a FIB-4 score $<1.45$ and a FIB-4 score $>3.25$ as F0-F1 (none or mild fibrosis) and F3-F4 (advanced fibrosis or cirrhosis), respectively. A total of 111 patients $(12 \%)$ had advanced fibrosis or cirrhosis [3]. The patients were classified into two groups: the "easy-to-treat" 758 patients and the "difficult-to-treat" 188 patients. The "difficult-to-treat" group included all 111 patients with advanced fibrosis or cirrhosis, as well as all 99 treatmentexperienced patients (including 77 non-cirrhotic patients): interferon- $\alpha$ /ribavirin, 16; SOF/interferon- $\alpha /$ ribavirin, 32; or SOF/simeprevir (SMV), 51 [3]. The "difficult-to-treat" or "easy-to-treat" group, respectively, was treated with a dual therapy of SOF/DCV with or without ribavirin daily for 12 weeks. SVR12 rates were achieved by 92\% (173/ $188)$ and $95 \%(718 / 758)$ of patients in the "difficult-totreat" and "easy-to-treat" groups, respectively. The main adverse events during and after 12 weeks of treatments were fatigues, asthenia, headache, gastrointestinal trouble, nausea and dyspnea. Serious adverse events were reported in seven patients (hospitalized due to four abdominal troubles and three anemia), and no cases of death or discontinuation were reported due to serious adverse events [3].

The authors also performed a resistance analysis in four relapsers, demonstrating, in two of four patients, a preexistence of the HCV NS5A-L28 M or NS5A-L30R polymorphism in association with DCV resistance at baseline and at the time of relapse, as well as no $\mathrm{HCV}$ NS5B-S282T variants in association with SOF resistance. Examination of baseline resistance-associated substitutions may be helpful to treat higher-risk groups like decompensated cirrhosis and treatment failures. These researchers concluded that the 12-week combination of SOF/DCV with or without ribavirin could achieve higher SVR rates with fewer adverse events in HCV GT4 Egyptian patients [3].

This study [3] also demonstrated that combinations of SOF/DCV with or without ribavirin for 12 or 24 weeks was effective and safe for chronic HCV GT4 "real-world" patients and supported that this regimen is one of the better options for patients with HCV GT4 infection and advanced liver disease [8]. The excellent SVR rates achieved by SOF/DCV [2, 3] have been prompting HCV eradication campaigns in Egypt.

Beginning in November 2015, generic SOF/DCV with or without ribavirin became the main therapy in the national program of Egypt due to cost-savings of more than $80 \%$ of the reduced cost of brand medications that were being used in the program [4]. Generic SOF/DCV with or without ribavirin could lead to higher SVR rates (95 or $95 \%$, respectively). In general, generic SOF/DCV, LDV/
SOF, or SOF/ribavirin showed excellent SVR rates [4]. In Egypt, generic versions of SOF/DCV have also played a role in the eradication of HCV GT4.

Both hypothyroidism and thyroid autoimmunity are more common in patients with chronic HCV infection [12]. Eletreby et al. [13] reported that 21\% (2833/13402) of HCV GT4 patients had an elevated thyroid-stimulating hormone $(\mathrm{TSH})$ level $>4.5 \mathrm{mIU} / \mathrm{L}$ (hypothyroidism) and $3.5 \%(466 / 13402)$ a low TSH level $<0.4 \mathrm{mIU} / \mathrm{L}$ (hyperthyroidism). A more advanced age and female gender were the only significant predictors of hypothyroidism by multivariate analysis. Interferon- $\alpha$ was associated with the development of thyroid autoimmunity and/or thyroid dysfunction in $5-12 \%$ of patients with chronic hepatitis $\mathrm{C}$, usually by unmasking subclinical abnormalities [12]. Pretreatment TSH did not affect SVR rates after DAA treatment including SOF/DCV with or without ribavirin [6, 13]. Moreover, thyroid dysfunction was improved in $80 \%$ of HCV GT4 patients with thyroid dysfunction who were being treated with DAAs [13].

AASLD and EASL HCV guidelines recommend pangenotypic regimens including $\mathrm{HCV}$ NS5A inhibitor velpatasvir or alternative regimen including $\mathrm{HCV} \mathrm{NS3/4A}$ inhibitor grazoprevir/HCV NS5A inhibitor elbasvir in HCV GT4 patients [9, 10]. Of course, these new regimens play an important role in treatment for special population such as patients with DAA-treatment failure. Generic SOF/ DCV preparations seem to be enough for treatment-naïve HCV GT4 Egyptian patients to be cured as SVR rates are higher with generic SOF/DCV preparations.

Once identified, HCV RNA-positive individuals should be linked to the treatment of SOF/DCV. After HCV RNA should be examined at week 24 after the end of treatment to confirm SVR, patients with SOF/DCV treatment failure may need alternative options. Nationwide treatment strategies using SOF/DCV with reduced cost may be useful for the eradication of HCV from Egypt.

In conclusion, the 12-week combination of SOF/DCV could result in higher SVR rates in HCV GT4 patients. In HCV GT4 patients with advanced liver fibrosis, the 24-week combination of SOF/DCV with ribavirin might also be useful. We should also focus on the generic versions of SOF/DCV. Furthermore, thyroid dysfunction was observed in $25 \%$ of HCV GT4-infected patients. However, interferon-free regimens including SOF/DCV with or without ribavirin, did not affect thyroid function or improve thyroid dysfunction. Attention should be paid to the long-term effects of interferon-free combinations of DAAs on thyroid function in clinical practice.

Funding None. 


\section{Compliance with ethical standards}

Conflict of interest Dr. Tatsuo Kanda received research grants from Merck Sharp and Dohme (MSD), Chugai Pharm and AbbVie. The funding sponsors played no role in the study design, data collection, analyses, interpretation, writing of the manuscript, or in the decision to publish the results. Shunichi Matsuoka and Mitsuhiko Moriyama declared that there are no conflicts of interest.

Ethical approval This article does not contain any studies with human participants or animals performed by any of authors.

\section{References}

1. Omata M, Kanda T, Yokosuka O, Crawford D, Al-Mahtab M, Wei $\mathrm{L}$, et al. Features of hepatitis $\mathrm{C}$ virus infection, current therapies and ongoing clinical trials in ten Asian Pacific countries. Hepatol Int 2015;9:486-507. https://doi.org/10.1007/ s12072-015-9630-4 PMID: 25941137.

2. Shiha G, Soliman R, ElBasiony M, Hassan AA, Mikhail NNH. Sofosbuvir plus Daclatasvir with or without ribavirin for treatment of chronic HCV genotype 4 patients: real-life experience. Hepatol Int 2018. https://doi.org/10.1007/s12072-018-9861-2 [Epub ahead of print] (PMID: 29663115).

3. Abdel-Moneim A, Aboud A, Abdel-Gabaar M, Zanaty MI, Ramadan M. Efficacy and safety of sofosbuvir plus daclatasvir with or without ribavirin: large real-life results of patients with chronic hepatitis C genotype 4. Hepatol Int 2018. https://doi.org/ 10.1007/s12072-018-9868-8 [Epub ahead of print] (PMID: 29754329).

4. Omar H, El Akel W, Elbaz T, El Kassas M, Elsaeed K, El Shazly $\mathrm{H}$, et al. Generic daclatasvir plus sofosbuvir, with or without ribavirin, in treatment of chronic hepatitis $\mathrm{C}$ : real-world results from 18378 patients in Egypt. Aliment Pharmacol Ther 2018;47:421-31. https://doi.org/10.1111/apt.14428 PMID: 29193226.

5. Shiha G, Esmat G, Hassany M, Soliman R, Elbasiony M, Fouad $\mathrm{R}$, et al. Ledipasvir/sofosbuvir with or without ribavirin for 8 or
12 weeks for the treatment of HCV genotype 4 infection: results from a randomised phase III study in Egypt. Gut 2018. https://doi. org/10.1136/gutjnl-2017-315906 [Epub ahead of print] (PMID: 29666174).

6. Ahmed OA, Kaisar HH, Badawi R, Hawash N, Samir H, Shabana SS, et al. Efficacy and safety of sofosbuvir-ledipasvir for treatment of a cohort of Egyptian patients with chronic hepatitis C genotype 4 infection. Infect Drug Resist 2018;11:295-8. https:// doi.org/10.2147/IDR.S153060 PMID: 29535545.

7. Gao M, Nettles RE, Belema M, Snyder LB, Nguyen VN, et al. Chemical genetics strategy identifies an HCV NS5A inhibitor with a potent clinical effect. Nature 2010;465:96-100. https://doi. org/10.1038/nature08960 PMID: 20410884.

8. Omata M, Kanda T, Wei L, Yu ML, Chuang WL, Ibrahim A, et al. APASL consensus statements and recommendation on treatment of hepatitis C. Hepatol Int 2016;10:702-26. https://doi. org/10.1007/s12072-016-9717-6 PMID: 27130427.

9. AASLD-IDSA. HCV guidance: recommendations for testing, managing, and treating hepatitis C. https://www.hcvguidelines. org/. Accessed 6 Jan 2016.

10. European Association for the Study of the Liver. EASL recommendations on treatment of hepatitis C 2018. J Hepatol 2018. https://doi.org/10.1016/j.jhep.2018.03.026 [Epub ahead of print] (PMID: 29650333).

11. Kanda T, Yokosuka O, Imazeki F, Tanaka M, Shino Y, Shimada $\mathrm{H}$, et al. Inhibition of subgenomic hepatitis $\mathrm{C}$ virus RNA in Huh-7 cells: ribavirin induces mutagenesis in HCV RNA. J Viral Hepat 2004;11:479-87. https://doi.org/10.1111/j.1365-2893.2004. 00531.x PMID: 15500548.

12. Antonelli A, Ferri C, Pampana A, Fallahi P, Nesti C, Pasquini M, et al. Thyroid disorders in chronic hepatitis C. Am J Med 2004;117:10-3. https://doi.org/10.1016/j.amjmed.2004.01.023 PMID: 15210382

13. Eletreby R, Said M, Abdellatif Z, Saad Y, ElSerafy M, Dabes H, et al. High prevalence of HCV (GT4)-related TSH abnormality among 13402 Egyptian patients treated with direct acting antiviral therapy. Hepatol Int 2018;12(2):143-148. https://doi. org/10.1007/s12072-018-9845-2 PMID: 29445911. 